\title{
Importância da barreira epidérmica na dermatite atópica canina: Revisão
}

\author{
Wendie Roldán Villalobos ${ }^{1 *}$ \& Laureano Rodríguez Beltrán ${ }^{2}$ \\ ${ }^{1}$ Médica Veterinária (Universidade de La Salle-Colômbia). Esp. (Universidade Federal do Rio Grande do Sul), MSc. \\ (Universidade Autónoma de Barcelona). Prática privada em Dermatologia Veterinária. Sócio pleno da SLDV (Sociedade \\ Latino-americana de Dermatologia Veterinária).E-mail: wendyrol21@yahoo.com \\ ${ }^{2}$ Médico Veterinário(Universidade Nacional de Colômbia). Docente Pós-graduação (Universidade CES -Colômbia). Prática \\ privada em Dermatologia Veterinária. Sócio fundador e pleno da SLDV. Sócio ativo da SBDV (Sociedade Brasileira de \\ Dermatologia Veterinária) e da SEDV (Sociedade Europeia de Dermatologia Veterinária. E-mail: \\ rodriguezblaureano@gmail.com \\ *Autor para correspondências
}

RESUMO. A dermatite atópica canina (D-AC) é uma doença alérgica, crônica e inflamatória da pele que afeita o 10-15\% dos cães. A patogênese da doença tinha girado em torno a processos de hipersensibilidade mediada pela $\mathrm{IgE}$, mas, nos últimos tempos, pesquisadores têm dirigido seus esforços à demonstração da importância da barreira epidérmica no desenvolvimento da DAC, baseando-se em sua maioria em estudos prévios da Dermatite Atópica Humana. Pesquisas relacionadas com a perda transepidérmica de água, os níveis de hidratação da pele e as anormalidades nos lipídeos do estrato córneo e nas proteínas superficiais da epiderme, principalmente na filagrina, têm sido fundamentais para avançar na compreensão da patogenia da DAC.

Palavras chave: Cães, barreira epidérmica, estrato córneo, dermatite atópica.

\section{Importance of epidermal barrier in canine atopic dermatitis: Review}

\begin{abstract}
Canine Atopic Dermatitis (CAD) is an allergic, chronic and inflammatory skin disease which affects $10-15 \%$ of canine population. For several years, pathogenesis of the disease had been linked to hypersensitivity processes mediated by IgE, but during the last decade, researchers have led their efforts to demonstrate the importance of the skin barrier in the development of CAD, based most of the times on previous studies related with Human Atopic Dermatitis. Studies in dog sincluding transepidermal water loss, skin moisture levels and abnormalities in the stratum corneum lipids and in the superficial proteins of the epidermis, filaggrin mainly, have been very important to advance the understanding of this common and frequent disease in veterinary medicine.
\end{abstract}

Keywords: Dogs, epidermal barrier, stratum corneum, atopic dermatitis.

\section{Introdução}

A dermatite atópica canina (DAC) é uma doença alérgica, crônica e inflamatória da pele, na qual existe uma predisposição genética (Ferrer, 2005) e afeita o 10-15\% da população canina (MacDonald, 2006). Prurido e eritema são considerados os sinais clínicos mais caraterísticos da DAC, apresentando-se principalmente em animais entre 6 meses e 3 anos de idade (Randall, 2005). Embora a patogênese da DAC tenha sido relacionada frequentemente com reações de hipersensibilidade mediadas pela Ig E
(Imunoglobulina E), na atualidade a evidência inclina-se a teorias que involucram a integridade da barreira epidérmica como fator fundamental no desenvolvimento da doença (Olivry et al,2010). A DAC comparte muitas similitudes clinicas e imunológicas com a Dermatite Atópica Humana (DAH) (Marsella \& Olivry, 2003), sendo principalmente a distribuição similar das lesões e a rota epicutânea da exposição alergênica (Marsella et al, 2006a; Olivry et al 2006; Marsella et al, 2006b) os fatores que incentivam a 
pesquisa das possíveis anormalidades presentes na barreira cutânea dos cães atópicos (Marsella et al, 2011).

\section{A barreira epidérmica}

Nos mamíferos, a pele é um órgão dinâmico que pode adaptar-se constantemente a câmbios no ambiente e tem importantes funções fisiológicas, imunológicas, sensoriais e de proteção. A epiderme da pele é composta pelos queratinócitos, que são submetidos a um processo de maturação bem organizado que leva finalmente à descamação (Hester et al, 2004).

O estrato córneo (EC) é a camada mais superficial da epiderme e é formada pelos corneócitos, ou queratinócitos cornificados, que estão rodeados pelos lipídeos lamelares complexos. O EC é fundamental na proteção da barreira cutânea, prevenindo a perda de agua transepidérmica e evitando o ingresso de sustâncias exógenas (Madison, 2003; Elias \& Schmuth, 2009). Além dos lipídeos, as proteínas são muito importantes nos processos de diferenciação e cornificação da epiderme (Marsella et al, 2011).

\section{Perda de água transepidérmica e hidratação da pele}

A integridade da barreira epidérmica nos humanos pode ser medida de uma forma não invasiva, através da perda de água transepidérmica ou TEWL (Transepidermal Water Loss) (Oestmann, 1993; Pinnagoda et al, 1990) que geralmente é feita utilizando evaporimetros de câmara aberta ou fechada (Olivry, 2011). A TEWL permite estabelecer a habilidade da pele para reter a humidade, e é medida em $\mathrm{g} / \mathrm{h} / \mathrm{m}^{2}$. A perda de água na pele ocorre principalmente por difusão passiva através da epiderme, é assim que valores elevados de TEWL indicam uma grande perda de água e são consistentes com danos na função da barreira do estrato córneo (Hester et al, 2004). O aumento dos níveis de TEWL tem relação direta com a intensidade da DA (Gupta et al, 2008; Hon et al, 2008), portanto a TEWL é maior em pacientes com DA (Loden et al, 1992; Lee et al, 2006; Seidnari \& Giusti, 1995; Boralevi et al, 2008) do que nos sujeitos controle sadios, inclusive em áreas não lesadas (Werner \& Lindberg et al, 1985). Nos humanos, a TEWL pode variar dependendo da localização anatômica (Kim et al, 2006; Nicander et al, 1997; Yosipovitch et al, $\underline{2000})$ e de outros fatores como a idade (Nicander et al, 1997; Wihelm et al, 1991). A utilidade da medição da TEWL em cães não é clara, devido a que existe uma variabilidade muito grande entre indivíduos e localizações anatômicas, que não permite fazer comparações confiáveis entre estudos clínicos (Beco \& Fontaine, 2000; Lau Gillard et al, 2010). A pesar das limitações, tem estudos que tentaram medir a TEWL com evaporimetros de câmara fechada em cães com DAC, em pele lesada e não lesada, e em cães sadios em locais similares, obtendo valores maiores nos animais com DAC (Shimada et al, 2009).Também existem estudos que utilizaram evaporimetros de câmara aberta em cães sensibilizados experimentalmente a ácaros do pó. Neste caso, os valores de TEWL foram maiores também nos cães com DAC induzida, particularmente nas localizações prediletas da doença, quando comparados com os cães normais (Hightower et al, 2010).

Outro método para medir a função da barreira cutânea é a determinação dos níveis de hidratação da pele, usando uma técnica conhecida como corneometria (Blichmann et al, 1988), que determina a capacitância da pele devido a seu comportamento como meio dielétrico (Hester et al, 2004). A água tem maior constante dielétrica do que a pele, e um aumento do conteúdo hídrico eleva os valores de capacitância, ou seja, a capacidade de guardar um gradiente de carga elétrica. $\mathrm{O}$ aparelho que permite essa medida baseia-se nas mudanças de tais constantes dielétricas, que variam, por conseguinte, a capacitância (Addor \& Aoki, 2010). Embora os valores obtidos na corneometria em cães em diferentes estudos tiveram variações entre indivíduos e localizações anatômicas, os resultados poderiam ser reproduzíveis para pesquisas futuras (Hester et al, 2004). Os níveis de hidratação da pele parecem ser similares entre a pele normal e a pele não lesada de cães com DAC. A pele lesada tem valores menores de humidade (Shimada et al, 2009).

\section{Lipídeos do estrato córneo}

Os lipídeos do EC são compostos principalmente por ácidos graxos livres, colesterol e ceramidas. As ceramidas constituem o 50\% dos lipídeos do estrato córneo nos humanos (Masukawa et al, 2009) e são os mais heterogêneos, incluindo 11 moléculas diferentes (Masukawa et al, 2008). As ceramidas são geradas de uma base esfingoide e um ácido graxo. Alguns exemplos de bases esfingoides 
incluem esfingosina, dihidroesfingosi- na e fitoesfingosina (Mizutani et al, 2009). A síntese das ceramidas efetua-se no estrato basal. Estas são rapidamente convertidas emglucosilceramidas e esfingomielinas que logo são agrupadas nos corpos lamelares (Ohnishi et al, 1999). Os corpos lamerales migram à membrana apical das células no estrato granuloso e liberam seus conteúdos nos espaços intercelulares. Os lipídeos excretados organizam-se em forma de bicamadas lipídicas superpostas (Holleran et al, 1993; MaoQiang et al, 1995a). Os níveis de ceramidas no EC são regulados através de um balance entre enzimas sintéticas e ceramidasas. Estas últimas podem ser endógenas ou exógenas (Ex: de bactéria) (Ohnishi et al, 1999).

O EC da pele dos pacientes humanos com DA é caracterizado pela redução na quantidade de ceramidase de aminoácidos hidrossolúveis (Macheleidt et al, 2002). Estudos realizados em cães com DAC, em zonas clinicamente não lesadas, demostraram que a morfologia dos lipídeos lamelares intercelulares nestes animais era mais heterogênea quando comparada com peles de cães normais. Adicionalmente, muitas áreas do EC atópico careciam de lipídeos lamelares e quando presentes possuíam uma estrutura anormal e/ou incompleta (Inman et al, 2001). Em cães sensibilizados experimentalmente, os desafios com ácaros do pó empioraram as anormalidades dos lipídeos do EC (Marsella et al, 2010). A redução na quantidade de ceramidas totais e de algumas subclasses de ceramidas livres e ligadas a proteí- nas em pacientes caninos atópicos com EC não lesados, é associada a um incremento na TEWL (Popa et al, 2011). Além disso, existe uma diminuição nos produtos da degradação das ceramidas antiinflamatórias S1P (Esfingosina-1-fosfato) na pele e no plasma, devida possivelmente a um aumento na rata de transcrição da sua enzima degradadora, a S1P liasa (Bäumer et al, 2011).

\section{Proteínas da epiderme}

A proteína que tem sido mais discutida na patogênese da DA nos últimos tempos é a filagrina (Marsella et al, 2011). Nos humanos, tem estudos que reportam uma diminuição da filagrinanos pacientes atópicostanto em peles lesadas quanto não lesadas (Seguchi et al, 1996). A filagrina é responsável por agregar a queratina e outras proteínas nas camadas mais superficiais da epiderme para a formação do EC (Tsai et al, 2006). O processo de conversão da profilagrina em filagrina mantém a integridade da epiderme (Addor \& Aoki, 2010). Nos humanos, tem sido candidatados variedade de genes que poderiam desenvolver a DA, mas, o gene que codifica a filagrina (FLG) é considerado o mais importante, devido a que suas mutações podem representar um risco maior para a aparição da DA (Baurecht et al, 2007; Palmer et al, 2006; O’Regan et al, $\underline{2010)}$.

Têm sido feitos três estudos que pesquisaram a expressão da filagrina nas peles de cães com DA e em cães sensibilizados experimentalmente. No ano 2009, analisaram-se biopsias de beagles com DA experimental por meio de imunohistoquimica, utilizando um antissoro monoclonal contra filagrina humana, mas, a tinção reconhecia, além da filagrina, outras proteínas como as queratinas, situação que limitou a validez dos resultados (Marsella et al, 2009). No 2010, foi efetuada a técnica de imunofluorescência indireta em peles de cães sadios e em peles não lesadas com DAC utilizando anticorpos específicos contra os extremos carboxi e amino da filagrina. Os resultados mostraram redução na expressão da filagrina, que segundo os autores, poderia ser secundaria a inflamação produzida na DA ou a mutações no gene FLG (Chervet et al, 2010). Outro estudo do ano 2010 pesquisou a expressão da filagrina em cães normais e sensibilizados experimentalmente a ácaros do pó, antes e depois do desafio alergênico. Após o desafio, a imunohistoquímica da proteína foi normal na epiderme dos cães sadios. Nos cães hipersensíveis, a tinção foi muito irregular, mas, após os sinais clínicos foram resolvidos, aumentou a imuno-reatividade da filagrina. Estes resultados sugerem que a inflamação reduz a expressão da filagrina só de maneira transitória (Santoro et al, 2010).

\section{Medidas terapêuticas para recuperar ou proteger a integridade da barreira epidérmica}

A eficácia clinica do uso tópico de lipídeos cutâneos complexos no tratamento da DAC tem sido objeto de pesquisa durante os últimos anos. Estudos prévios em cães têm mostrado câmbios significativos, químicos e estruturais, no EC após 3 semanas de tratamento tópico com uma emulsão contendo ceramidas, ácidos graxos livres e colesterol (Piekutowska et al, 2008; Popa et al, 2012) enquanto que num outro estudo os benefícios foram vistos após 4 semanas de aplicação (Marsella et al, 2012). 
Os shampoos contendo fitoesfingosina ou combinações de antissépticos, ácidos graxos e açúcares complexos têm sido testados em cães com DAC, mostrando uma diminuição no grado de prurido e no CADESI (Canine Atopic Dermatitis Extentand Severity Index) (Bourdeau et al, 2007). Outro estudo que pretendia provar a eficácia de produtos tópicos com óleos essenciais de plantas e ácidos graxos não saturados (spot-on e spray) obteve resultados similares, com redução nos níveis de prurido e no CADESI, mas, sem evidência nenhuma de melhora nos valores da TEWL (Tretter \& Mueller, 2011).

Além disso, existem protetores cutâneos que agem como uma barreira exógena que pretende evitar a perda de água e reter a humidade no EC (Mao-Qiang, 1995b; Ghadially et al, 1992). A dimeticona é um tipo de óleo de silicone com propriedades que fazem que seja um ingrediente muito útil em diversos produtos de cuidado pessoal e é comumente utilizada para reparar a pele lesada nos humanos. Segundo a FDA (Food and Drug Administration), concentrações entre 1$30 \%$ são consideradas seguras como protetor cutâneo (Pellicoro et al, 2013). Em humanos, tem sido demostrado que o uso rotineiro de emolientes tópicos pode diminuir a necessidade de terapia com glucocorticoóides tópicos (Lucky et al, 1997). Embora estudos realizados em cães não mostras sem benefícios clínicos com o uso da dimeticona, esta poderia ser uma alternativa inovadora no futuro como terapia adjunta na DAC (Pellicoro et al, 2013).

É importante ressaltar que a utilização de este tipo de produtos deveria ser combinada com fármacos anti-inflamatórios e antimicrobianos além da imunoterapia, objetivando uma aproximação global à doença (Fujimura et al, 2011).

A atualização das recomendações no tratamento da DAC do ano 2015 publicada pelo ICADA (International Committee on Allergic Diseases of Animals) cita em alguns espaços os possíveis benefícios dos AGE nos casos crônicos da DAC. A administração oral de ácidos graxos essenciais, especialmente aqueles ricos em Omega 6, como suplemento ou incluídos em dietas comerciais, podem influenciar positivamente aos lipídeos de superfície melhorando o brilho e a qualidade da pelagem além de reduzir os sinais clínicos da DAC, embora não sejam recomendados como terapia única. Os possíveis resultados do uso dos AGE não seriam observados antes de dois meses de suplementação. Em geral, as dietas enriquecidas com AGE fornecem maiores quantidades destes do que os suplementos orais. As formulações tópicas poderiam ajudar na normalização dos defeitos da barreira cutânea em cães com DAC. $\mathrm{O}$ uso concomitante de imunoterapia alérgenoespecífica, shampoos emolientes e AGE, em dietas ou suplementação oral, permitiriam a diminuição das doses e/ou a frequência dos glucocorticoides orais, da ciclosporina e do oclacitinib requeridas para manter a remissão dos sinais clínicos em pacientes com DAC crónica (Olivry et al, 2015).

\section{Considerações finais}

A Dermatite Atópica Canina (DAC) é uma doença alérgica comum e de grande importância em dermatologia veterinária devido a sua natureza crônica e recidivante. A patogênese da DAC tem sido objeto de estudo por décadas, e atualmente existem achados que têm permitido determinar a importância da barreira epidérmica no desenvolvimento da patologia. Estudos que involucram a perda transepidérmica de água, os níveis de hidratação da pele e as anormalidades nos lipídeos do estrato córneo e nas proteínas da epiderme, têm aberto ainda mais o caminho para o entendimento da doença. Ainda continua sendo necessário estabelecer qual a forma adequada de reparar a barreira cutânea lesada além de confirmar os possíveis benefícios das opções terapêuticas conhecidas até hoje.

\section{Referências Bibliográficas}

Addor, F. \& Aoki V. (2010). Barreira cutânea na dermatite atópica. Anais Brasileiros de Dermatologia. 2: 184-94.

Bäumer, W., Rossbach, K., Mischke, R., Reines, I., Langbein-Detsch, I., Lüth, A. \& Kleuser, B. (2011). Decreased concentration and enhanced metabolism of sphingosine-1phosphate in lesional skin of dogs with atopic dermatitis: disturbed sphingosine-1-phosphate homeostasis in atopic dermatitis. Journal of Investigative Dermatology. 131:266-268.

Baurecht, H., Irvine A., Novak N., Illig, T., Bühler, B., Ring, J., Wagenpfeil, S. \& Weidinger S. (2007). Toward a major risk factor for atopic eczema: meta-analysis of filaggrin polymorphism data. Journal of Allergy and Clinical Immunology. 120: 140612. 
Beco, L. \& Fontaine, J. (2000). Corneometry and transepidermal water loss measurements in the canine species: validation of these techniques. Annales de MédecineVétérinaire. 144: 329-333.

Blichmann, C. \& Serup, J. (1988) Assessment of skin moisture. Measurement of electrical conductance, capacitance and transepidermal water loss. Acta Dermato-Venereologica. 68: 284-290.

Boralevi, F., Hubiche, T., Leaute-Labrze, C., Saubusse, E., Fayon, M., Roul, S., MauriceTison, S. \& Taïeb, A. (2008) Epicutaneous aeroallergen sensitization in atopic dermatitis infants - determining the role of epidermal barrier impairments. Allergy. 63: 205- 10.

Bourdeau, P., Bruet, V., Gremillet, C. (2007). Evaluation of phytosphingosine-containing shampoo and microemulsion spray in the clinical control of allergic dermatoses in dogs: preliminary results of a multicentre study (abstract). Veterinary Dermatology. 18: 177178.

Chervet, L., Galichet, A., McLean, W., Chen, H., Suter, M., Roosje, P. \& Müller, E. (2010). Missing C-terminal filaggrin expression, NFkappaB activation and hyperproliferation identify the dog as a putative model to study epidermal dysfunction in atopic dermatitis. Experimental Dermatology. 19: e343-e346.

Elias, P. \& Schmuth, M. (2009). Abnormal skin barrier in the etiopathogenesis of atopic dermatitis. Current Opinion in Allergy and Clinical Immunology. 9: 437-446.

Ferrer, L. (2005). Canine atopic dermatitis: evidence based dermatology. In: Proceedings of the North American Veterinary Conference. January 8-12, 2005. Florida, United States. 244-246.

Fujimura, M., Nakatsuji, Y., Fujiwar, S., Rème, C. \& Gatto, H. (2011). Spot-On Skin Lipid Complex as an Adjunct Therapy in Dogs with Atopic Dermatitis: An Open Pilot Study. Veterinary Medicine International, ID 281846. Doi:10.4061/2011/2818 46

Ghadially, R., Halkier-Sorensen, L. \& Elias, P. (1992). Effects of petrolatum on stratum corneum structure and function. Journal of the American Academy of Dermatology, 26 (3): 387-396.
Gupta, J., Grube, E., Ericksen, M., Stevenson, M., Lucky, A., Sheth, A., Assa'ad, A. \& Khurana - Hershey, G. (2008). Intrinsically defective skin barrier function in children with atopic dermatitis correlates with disease severity. Journal of Allergy and Clinical Immunology. 121: 725-30.

Hester, S., Rees, C., Kennis, R., Zoran, D., Bigley, K., Wright, A., Kirby, N. \& Bauer, J. (2004). Evaluation of Corneometry (Skin Hydration) and Transepidermal Water-Loss Measurements in Two Canine Breeds. Journal of Nutrition. 134: 2110S-2113S.

Hightower, K., Marsella, R., Flynn-Lurie, A. (2010). Effects of age and allergen exposure on transepidermal water loss in a house dust mite-sensitized beagle model of atopic dermatitis. Veterinary Dermatology. 21: 8895.

Holleran, W., Takagi, Y., Menon, G.,Legler, G.,Feingold, K. \& Elias, P. (1993). Processing of epidermalglucosyl- ceramides is required for optimal mammalian permeability barrier function. Journal of Clinical Investigation. 91: 1656e-1664.

Hon, K., Wong, K., Leung, T. \& Chow, C.,Ng, P. (2008). Comparison of skin hydration evaluation sites and correlations among skin hydration, transepidermal water loss, SCORAD index, Nottingham Eczema Severity Score, and quality of life in patients with atopic dermatitis. American Journal of Clinical Dermatology. 9: 45-50

Inman, A., Olivry, T., Dunston, S., MonteiroRiviere, N. \& Gatto H. (2001). Electron microscopic observations of the stratum corneum intercellular lipids in normal and atopic dogs. Veterinary Pathology. 38: 720723.

Kim, D., Park, J., Na, G., Lee, S. \& Lee, W. (2006). Correlation of clinical features and skin barrier function in adolescent and adult patients with atopic dermatitis. International Journal of Dermatology. 45:698-701.

Lau-Gillard, P., Hill, P., Chesney, C., Budleigh, C. \& Immonen, A. (2010). Evaluation of a hand-held evaporimeter (VapoMeter) for the measurement of transepidermal water loss in healthy dogs. Veterinary Dermatology. 21: 136-145. 
Lee, C., Chuang, H., Shih, C.,Jong, S.,Chang, C. \& Yu, H. (2006). Transepidermal water loss, serum IgE and b-endorphin as important and independent biological markers for development of itch intensity in atopic dermatitis. British Journal of Dermatology. 154: 1100-7.

Loden, M., Olsson, H., Axell, T. \& Linde, Y. (1992). Friction, capacitance and transepidermal water loss (TEWL) in dry atopic and normal skin. British Journal of Dermatology. 126: 137-41.

Lucky, A., Leach, A., Laskarzewski, P. \& Wenck, H. (1997). Use of an emollient as a steroid-sparing agent in the treatment of mild to moderate atopic dermatitis in children. Pediatric Dermatology. 14(4):321-324.

MacDonald, J. (2006). Allergen specific immunotherapy for atopy. In: Proceedings of the North American Veterinary Conference. January 7-11, 2006. Florida, United States. 20: 396-398.

Macheleidt, O., Kaiser, H. \& Sandhoff, K. (2002). Deficiency of epidermal proteinbound $\mathrm{x}$-hydroxyceramides in atopic dermatitis. Journal of Investigative Dermatology. 119: 166-73.

Madison, K. (2003). Barrier function of the skin: la raison d'être of the epidermis. Journal of Investigative Dermatology. 121: 231-241.

Mao-Qiang, M., Feingold, K., Jain, M. \& Elias, P. (1995a). Extracellular processing of phospholipids is required for permeability barrier homeostasis. Journal of Lipid Research. 36: 1925-1935.

Mao-Qiang, M., Brown, B., Wu-Pong, S., Feingold, K. \& Elias, P. (1995b). Exogenous nonphysiologic vs physiologic lipids: divergent mechanisms for correction of permeability barrier dysfunction. Archives of Dermatology. 131(7):809-816.

Marsella, R. \& Olivry, T. (2003). Animal models of atopic dermatitis. Clinics in Dermatology. 21: $122-33$.

Marsella, R., Nicklin, C. \& Lopez, J. (2006a) Studies on the role of routes of allergen exposure in high IgE-producing beagle dogs sensitized to house dust mites. Veterinary Dermatology. 17: 306-12.
Marsella, R., Olivry, T. \& Maeda, S. (2006b). Cellular and cytokine kinetics after epicutaneous allergen challenge (atopy patch testing) with house dust mites in high-IgE beagles. Veterinary Dermatology. 17: 111-20.

Marsella, R., Samuelson, D. \& Harrington, L. (2009). Immunohistochemical evaluation of filaggrin polyclonal antibody in atopic and normal beagles. Veterinary Dermatology. 20: 547-555.

Marsella, R., Samuelson, D. \& Doerr, K., (2010). Transmission electron microscopic studies in an experimental model of canine atopic dermatitis. Veterinary Dermatology. 21: 8188.

Marsella, R., Olivry, T. \& Carlotti, D. (2011). Current evidence of skin barrier dysfunction in human and canine atopic dermatitis. Veterinary Dermatology. 22: 239-48.

Marsella, R., Genovese, D., Gilmer, L., Ahrens, K., Gatto, H. \& Navarro, C. (2012). Investigations on the effects of a topical ceramide and free fatty acid solution (Allerderm Spot on) on clinical signs and skin barrier function in dogs with atopic dermatitis: a double blinded, randomized, controlled study. In: Proceedings of the 7th World Congress of Veterinary Dermatology. Vancouver, Canada. 2012.

Masukawa, Y., Narita, H., Sato, H., Naoe, A., Kondo, N., Sugai, Y., Oba, T., Homma, R., Ishikawa, J., Takagi, Y. \& Kitahara, T. (2009). Comprehensive quantification of ceramide species in human stratum corneum. Journal of Lipid Research. 50: 1708-19.

Masukawa, Y., Narita, H., Shimizu, E., Kondo, N., Sugai, Y., Oba, T., Homma, R., Ishikawa, J., Takagi, Y., Kitahara, T., Takema, Y. \& Kita, K. (2008). Characterization of overall ceramide species in human stratum corneum. Journal of Lipid Research. 49: 1466-76.

Mizutani, Y., Mitsutake, S., Tsuji, K., Kihara, A. \& Igarashi, Y. (2009). Ceramide biosynthesis in keratinocyte and its role in skin function. Biochimie. 91:784-90.

Nicander, I., Nyren, M., Emtestam, L. \& Ollmar, S. (1997). Baseline electrical impedance measurements at various skin sites - related to age and sex. Skin Research and Technology. 3: $252-8$. 
Nikolovski, J., Stamatas, G., Kollias, N. \& Wiegand, B. (2008). Barrier function and water-holding and transport properties of infant stratum corneum are different from adult and continue to develop through the first year of life. Journal of Investigative Dermatology. 128: 1728-36.

Oestmann, E., Lavrijsen, A., Hermans, J. \& Ponec, M. (1993). Skin barrier function in healthy volunteers as assessed by transepidermal water loss and vascular response to hexyl nicotinate: intra- and interindividual variability. British Journal of Dermatology. 128: 130-6.

Ohnishi, Y., Okino, N., Ito, M. \& Imayama, S. (1999). Ceramidase activity in bacterial skin flora as a possible cause of ceramide deficiency in atopic dermatitis. Clinical and Diagnostic Laboratory Immunology. 6: 1014.

Olivry, T., Deangelo, K., Dunston, S., Clarke, K. \& McCall, C. (2006). Patch testing of experimentally sensitized beagle dogs: development of a model for skin lesions of atopic dermatitis. Veterinary Dermatology. 17: 95-102.

Olivry, T., De Boer, D., Favrot, C., Jackson, H., Mueller, R., Nuttall, T. \& Prélaud, P. (2010). Treatment of canine atopic dermatitis: 2010 clinical practice guidelines from the International Task Force on Canine Atopic Dermatitis. Veterinary Dermatology. 21(3):233-248.

Olivry, T. (2011). Is the skin barrier abnormal in dogs with atopic dermatitis?. Veterinary Immunology and Immunopathology. 144: 1116.

Olivry, T., DeBoer, D., Favrot, C., Jackson, H., Mueller, R., Nuttall, T. \& Prélaud, P. (2015). Treatment of canine atopic dermatitis: 2015 updated guidelines from the International Committee on Allergic Diseases of Animals (ICADA). Veterinary Research. 11(1):210. DOI 10.1186/s12917-015-0514-6

O’Regan, G. \& Irvine, A. (2010). The role of filaggrin in the atopic diathesis. Clinical and Experimental Allergy. 40: 965-72

Palmer, C., Irvine, A., Terron-Kwiatkowski, A., Zhao, Y.,Liao, H., Lee, S., Goudie, D, Sandilands A., Campbell, L., Smith, F., O'Regan, G., Watson, R., Cecil, J., Bale, S.,
Compton, J., Di Giovanna, J., Fleckman, P., Lewis-Jones, S., Arseculeratne, G., Sergeant, A., Munro, C., El Houate, B., Mc Elreavey, K., Halkjaer, L., Bisgaard, H., Mukhopadhyay, S. \& McLean, W. (2006). Common loss-of-function variants of the epidermal barrier protein filaggrin are a major predisposing factor for atopic dermatitis. Nature Genetics. 38: 441-6.

Pellicoro, C., Marsella, R. \& Ahrens, K. (2013). Pilot Study to Evaluate the Effect of Topical Dimethicone on Clinical Signs and Skin Barrier Function in Dogs with Naturally Occurring Atopic Dermatitis. Veterinary Medicine International. ID 239186. http://dx.doi.org/10.1155/2013/239186.

Piekutowska, A., Pin, D., Rème, C., Gatto, H. \& Haftek, M. (2008). Effects of a topically applied preparation of epidermal lipids on the stratum corneum barrier of atopic dogs. Journal of Comparative Pathology. 138: 197203.

Pinnagoda, J., Tupker, R., Agner, T. \& Serup, J. (1990). Guidelines for transepidermal water loss (TEWL) measurement. A report from the standardization group of the European society of contact dermatitis. Contact Dermatitis. 22: 164-78.

Popa, I., Remoue, N., Hoang, L., Pin, D., Gatto, H., Haftek, M. \& Portoukalian, J. (2011). Atopic dermatitis in $\operatorname{dogs}$ is associated with a high heterogeneity in the distribution of protein-bound lipids within the stratum corneum. Archives of Dermatological Research. 303: 433-440.

Popa, I., Remoue, N., Osta, B., Pin, D., Gatto, H., Haftek, M. \& Portoukalian, J. (2012). The lipid alterations in the stratum corneum of dogs with atopic dermatitis are alleviated by topical application of a sphingolipidcontaining emulsion. Clinical and Experimental Dermatology. 37(6): 665-671.

Randall, C. (2005). Canine atopic dermatitis: old and new therapies. In: Proceedings of the North American Veterinary Conference. January 8-12. Florida, United States. 285-288.

Santoro, D., Marsella, R., Bunick, D., Graves, T., Campbell, K. \& Ahrens, K. (2010). Expression and distribution of canine filaggrin in the skin of healthy and atopic beagles (abstract). Veterinary Dermatology. 21: 323. 
Seidnari, S. \& Giusti, G. (1995). Objective assessment of the skin of children affected by atopic dermatitis: a study of $\mathrm{pH}$, capacitance and TEWL in eczematous and clinically normal skin. Acta Dermato-Venereologica. 75: 429-33.

Seguchi, T., Cui, C., Kusuda, S., Takahashi, M., Aisu, K. \& Tezuka, T. (1996). Decreased expression of filaggrin in atopic skin. Archivesof Dermatological Research. 288: 442-6.

Shimada, K., Yoon, J., Yoshihara, T., Iwasaki, T. \& Nishifuji, K. (2009). Increased transepidermal water loss and decreased ceramide content in lesional and non-lesional skin of dogs with atopic dermatitis. Veterinary Dermatology. 20: 541-546.

Tretter, S. \& Mueller, R.S. (2011). The influence of topical unsaturated fatty acids and essential oils on normal and atopic dogs. Journal of the American Animal Hospital Association. 47: 236-240.

Tsai, K., Valente, N. \& Nico, M. (2006). Inflammatory peeling skin syndrome studied with electron microscopy. Pediatric Dermatology. 23:488-92.
Werner, Y. \& Lindberg, M. (1985). Transepidermal water loss in dry and clinically normal skin in patients with atopic dermatitis. Acta Dermato-Venereologica. 65: $102-5$.

Wilhelm, K., Cua, A. \& Maibach, H. (1991). Skin aging. Effect of transepidermal water loss, stratum corneum hydration, skin surface $\mathrm{pH}$ and casual sebum content. Archives of Dermatology. 127: 1806-9.

Yosipovitch, G., Maayan-Metzger, A., Merlob, P. \& Sirota, L. (2000). Skin barrier properties in different body areas in neonates. Pediatrics. 106: $105-8$.

Recebido em Março 26, 2016

Aceito em Abril 22, 2016

License information: This is an open-access article distributed under the terms of the Creative Commons Attribution License, which permits unrestricted use, distribution, and reproduction in any medium, provided the original work is properly cited. 